\title{
Fingerprint Verification based on Fusion of Minutiae and Ridges using Strength Factors
}

\author{
Shashi Kumar D. R. \\ Department of CSE, Cambridge Institute of \\ Technology, Bangalore
}

\author{
K.B. Raja. \\ Department of ECE, University Visvesvaraya College \\ of Engineering, Bangalore University, Bangalore
}

\begin{abstract}
With the increasing emphasis on the automatic personal identification applications, biometrics especially fingerprint identification is the most reliable and widely accepted technique. In this paper Fingerprint Verification based on fusion of Minutiae and Ridges using Strength Factors (FVMRSF) is presented. In the preprocessing stage the Fingerprint is Binarised and Thinned. The Minutiae Matching Score is determined using Block Filter and Ridge matching score is estimated using Hough Transform. The strength factors Alpha $(\alpha)$ and Beta $(\beta)$ are used to generate Hybrid matching score for matching of fingerprints. The proposed algorithm has better matching percentage for different fingerprints compared to the existing algorithms.
\end{abstract}

\section{Keywords:}

Minutiae, Ridge, Dilation, Ridge Bifurcations, Block Filter, Hough Transform, Strength Factor

\section{INTRODUCTION}

The biometric recognition of a person refers to the use of certain physiological or behavioral features to determine the identity of a person. Traditionally passwords (knowledge-based security) and ID cards (token-based security) are used to access control to restricted systems or places. However, these systems are prone to fraud when a password is divulged to an unauthorized user or a card is stolen. Furthermore, simple passwords are easy to guess by an impostor, while difficult passwords may be hard to recall by a legitimate user. Biometrics has the capability to distinguish between an authorized user and imposter. The emergence of biometric technology has provided an attractive alternate to solve the problems present in traditional verification methods. Fingerprint is the most practical and widely used biometric technique in personal identification for several centuries. Among all the other kind of popular personal identification methods, fingerprint identification is the most mature and reliable technique.

Uniqueness and permanence are the two properties of fingerprint identification. It is claimed that no two individuals including identical twins have the same fingerprints and does not change throughout the lifetime, with the exception of a significant injury to the finger that creates a permanent scar. Fingerprint recognition takes advantage of the fact that the fingerprint has some unique characteristics such as minutiae and the ridges. The use of multibiometric systems is considered as one of the keys to improve the accuracy of biometric systems, than single biometric parameter and one matching algorithm.

\author{
R. K. Chhotaray \\ Department of CSE, Seemanta Engineering College, \\ Mayurbhanj, Orissa
}

Sabyasachi Pattanaik

Department of I\&CT

F.M. University, Balasore, Orissa

\begin{abstract}
Fingerprint technology is used by hundreds of thousands of people daily to access network PCs, PC/Network Access, Physical Security/Time, Attendance and Civil ID. Many military bases and government buildings use computers to check fingerprints of employees before they are admitted to secure areas. The fingerprint applications are divided into three categories (i) Forensic applications used for criminal investigation, terrorist identification, parenthood determination, missing children etc. (ii) Government applications, National ID cards, correction facility, Driving license, Passport Control etc. (iii) Commercial applications are knowledge based system such as Computer Network Logic, Electronic Data Security, E-commerce, Internet Access, ATM, Credit Cards, Cellular Phones, Personal Digital Assessment and Medical Record Management.
\end{abstract}

Contribution: In this paper minutiae and ridge based methods are simultaneously used in order to improve the matching performance and to reduce the false matching ratio considerably. In Minutiae based method we used block filter thinning method, where as in ridge based method the Hough transform is used to extract fingerprint features to compute hybrid matching score.

Organization: The paper is organized into the following sections. Section 2 is an overview of related work. The FVMRSF model is described in Section 3. Section 4 is the algorithm of FVMRSF system. Performance analysis of the system is presented in Section 5 and Conclusions are contained in Section 6.

\section{Related work}

Michael Kucken and Newell [1] discussed the hypothesis on the development of epidermal ridges viz., (i) the epidermal ridge pattern is established as a result of buckling instability acting on the basal layer of the epidermis and resulting in the primary ridges. (ii) The buckling process underlying fingerprint development is controlled by the stresses formed in the basal layer and not by the curvatures of the skin surface and (iii) the stresses that determine ridge direction are themselves determined by boundary forces acting at creases and nail furrow, normal displacements which are most pronounced close to the ridge. Ashish Mishra et. al., [2] proposed the algorithm based on orientation field of the fingerprint, over which gradient filter mask was used to detect the core points in the fingerprint image. Bazen and Gerez [3] presented methods for the estimation of a high resolution directional field from fingerprints. The directional field detects the singular points and the orientations of the points. Yun and Cho [4] proposed an adaptive preprocessing method, which extracts five features from the 
fingerprint images, analyzes image quality with clustering method, and enhances the images according to their characteristics. The preprocessing is performed after distinguishing the fingerprint image quality according to its characteristics.

Brankica M. Popovi'c and L. Maskovic [5] used multiscale directional information obtained from orientation field image to filter the spurious minutiae. The feature extraction in pattern recognition system is to extract information from the input data and depends greatly on the quality of the images. Multiscale directional information estimated based on orientation field estimation. F. A. Afsar et. al., [6] presented the minutiae based Automatic Fingerprint Identification Systems. The technique is based on the extraction of minutiae from the thinned, binarized and segmented version of a fingerprint image. The system uses fingerprint classification for indexing during fingerprint matching. G. Jagadeeswar Reddy et. al., [7] presented fingerprint denoising using both wavelet and Curvelet Transforms. The search-rearrangement method performs better than minutiae based matching for fingerprint binary constraint graph matching since implicit alignment of two fingerprint images are not required. K. Zebbiche and F. Khelifi [8] presented biometric images as one Region of Interest (ROI) that has the data processed by most biometric based systems. The scheme consists of embedding the watermark into ROI in fingerprint images. Discrete Wavelet Transform and Discrete Fourier Transform are used. Bhupesh Gour et. al., [9] introduced midpoint ridge contour representation in order to extract the minutiae from fingerprint images. Colour coding scheme is used to scan each ridge only once. Seung Hoon chae and Jong $\mathrm{Ku}$ Kim [10] proposed Fingerprint Verification in which both minutiae and ridge information are used to reduce the errors due to incomplete alignment or distortion. Aparecido Nilcau Marana and A. K. Jain [11] proposed Ridge Based Fingerprint matching using the Hough transform. The major straight lines that match the fingerprint ridges are used to estimate rotation and translation parameters.

Anil K Jain et al., [12] described the use of logistic regression method to integrate multiple fingerprint matching algorithms. The integration of Hough transform matching, string distance matching and 2D dynamic programming based matching using the logistic regression has minimized the False Rejection Rate for a specified level of False Acceptance Ratio. Fanglin Chen et al., [13] proposed an algorithm for reconstructing fingerprint orientation field from saved minutiae and is used in the matching stage to compare with the minutiae from the query fingerprint. The orientation fields computed from the saved minutiae is a global feature and the saved minutiae are the local feature, are used to get more information. Chunxian Ren et al., [14] used the hybrid algorithm based on linear classifier to segregate foreground and background blocks. The pixel wise classifier uses three pixel features such as Coherence, mean and variance. Hartwig Fronthaler et al., [15] proposed a multigrid representation of a discrete differential scale space enhancement strategy of fingerprint recognition system. The fingerprint image is decomposed using Laplacian Pyramid as relevant information is concentrated within a few frequency bands. The Fausian Directional Filtering is used to enhance ridge valley pattern of fingerprint using 1-D filtering on higher pyramid level. The linear symmetric features are used to extract the local ridge -valley orientation. Shabana Tadvi and Mahesh Kalte [16] proposed the method of combining the minutiae features and wavelet statistical features to obtain the hybrid fingerprint matching. Liu Wei and
Zhou Cong [17] presented Gradual Segmentation algorithm and multi segmentation features for fingerprint image segmentation. The fingerprint region is obtained using a Gradual Segmentation and recoverable region is segmented using Multi Segmentation feature algorithm.

Hemanth Krishnappa and Hongyu Guo [19] proposed fingerprint verification using mutual information. The input image is subjected to preprocessing using filtering and normalization which involves translation and Rotation. The mutual information at each step of Rotation and translation is calculated to find the best alignment of fingerprint by maximizing the mutual information. Swapnali Mahadik et al., [20] described an Alignment based Minutiae Matching algorithm. The minutiae extraction involves Filtering, Binarization, Orientation Estimation, Region of interest, Thinning and Minutiae Extraction. In the matching stage the images are subjected to translation, rotation and scaling.

Yi Chen and Anil K Jain [21] proposed an algorithm based on fingerprint features viz., minutiae and ridges, Pattern and Pores. The correlation among Fingerprint features and their distributions are considered for the model.Johg Ku Kum et al., [22] described a study on of Hybrid fingerprint matching methods. The minutiae and image based fingerprints verification methods are implemented together. The shapes in the fingerprint such as square, diamond, cross and dispersed cross are used for matching. Manvjeeth Kaur et al., [23] proposed fingerprint verification system adopting many methods to build a minutiae extractor and a minutiae matcher. The method with some changes like segmentation using morphological operation, thinning, minutiae marking with special triple branch counting, Minutiae unification by decomposing a branch into three terminations, matching the unified $\mathrm{x}-\mathrm{y}$ co-ordinate system are employed.Liu Wei [24] described Rapid Singularity Searching for fingerprint classification. The algorithm uses Delta Poincare Index and Rapid Classification to classify the fingerprint into five classes. The Singularity is achieved by detection algorithm which searches the direction field that has the larger directional change. Arun Ross et al., [25] proposed the hybrid fingerprint matcher which employs the combination of ridge strengths and a set of minutiae points. Zhang Yuanyuan and Jing Xianjun [26] presented the fingerprint image enhancement algorithm where the characteristics of the Gabor filter functions were analyzed both in spatial and spectral domain to obtain filter envelop parameters. These parameters were then convolved with the digital image to enhance the fingerprint image.

\section{MODEL}

In this section we discuss definitions and FVMRSF model.

\subsection{Definitions:}

(i) Fingerprint: Impression of a finger acquired from digital scanners.

(ii) Minutiae: Ridge bifurcations, Ridge endings in fingerprint image.

(iii) Ridge bifurcation: The ridge splits into two ridges.

(iv) Ridge termination: The ridge end point.

(v) False Minutiae: The points which are incorrectly identified as minutiae.

(vi) Pores: The patterns which are almost evenly placed along the ridges.

\subsection{Block Diagram of FVMRSF}




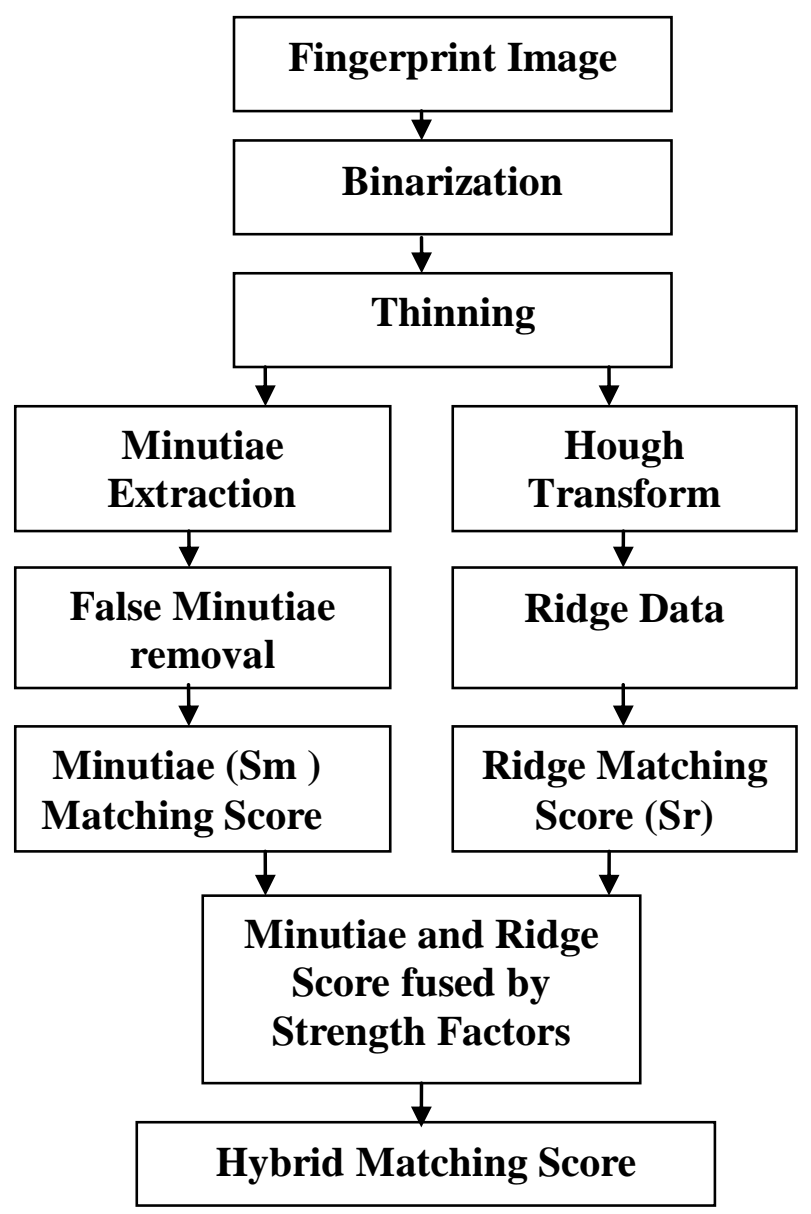

Fig 1. Block Diagram of FVMRSF.

\subsubsection{Fingerprint Image}

A gray-level fingerprint image (I) of size $640 * 480$ from the data base fvc2004 is considered.

\subsubsection{Binarization}

The process of converting a grayscale image to binary image is known as binarization. In a gray-scale fingerprint image, a pixel can take on 256 different intensity levels. The threshold value is used to convert grayscale image to binary. The pixel values below the threshold are set to zero and the intensity values greater than the threshold is assigned one. In binary image the pixel values are assigned 0 and 1 to black and white pixels respectively. The processing of binary image is easy as it has only two intensity levels compared to gray scale image of 256 intensity levels. Figure 2 shows original fingerprint image and its corresponding binarized image. The disadvantage of binarization is that the ridges end near the boundary is considered as minutia even though it is not actual minutia. The problem of binarization is eliminated in thinning process.

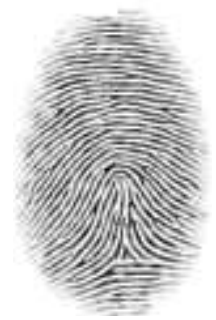

(a) Original image

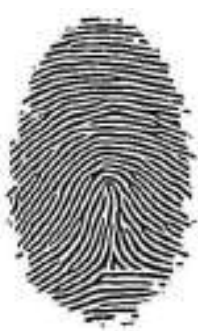

(b) Binarized image
Fig 2. Fingerprint Binarization

\subsubsection{Thinning:}

The thickness of ridge is converted into single pixel wide by keeping original orientation and location of the minutiae to ensure accurate estimation by thinning and is implemented using Block Filter. Dilation is used to thicken the area of the valleys in the fingerprint as a result the ridges are effectively eroded. A conservative structuring element consisting of four ones arranged in a two-by-two square is used for the valley dilation to achieve some ridge width reduction, while minimizing the discontinuities. Figure 3 gives binarized image and its corresponding valley dilation.

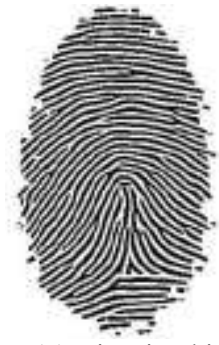

(a) Binarized image
Fig 3. Ridge Thinning.

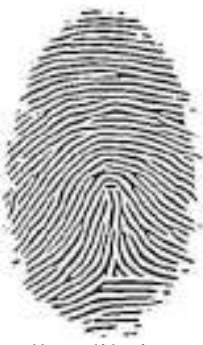

(b) Valley dilation

\subsubsection{Block Filtering}

The thinning method is to preserve the outermost pixels along each ridge. The border is fixed for fingerprint image to eliminate spur minutiae by assigning pixel values as one for first five rows, last five rows, first five columns and last five columns. The left to right scan for ridges that move up and right, while right to left scan for ridges that move down and left is used to reduce ridge width to one pixel. The two scans are combined to reduce ridge width to one pixel for whole fingerprint as shown in Figure 4.
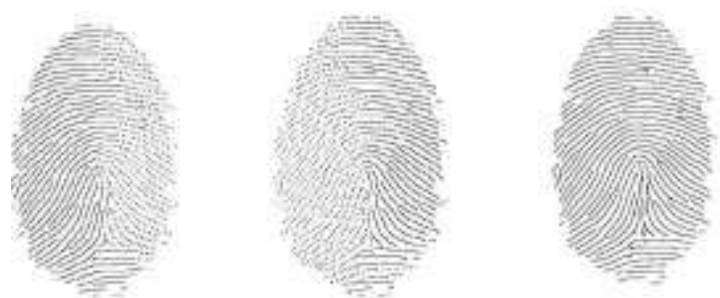

(a) Left to Right scan (b) Right to Left scan (c) Combined Fig 4. Scanned output of filter.

The isolated noise is eliminated using pixel box method by computing crossing number for each black pixel in the thinned fingerprint. The crossing number is half the sum of difference between adjacent pixel pairs that surrounded the given black pixel. 


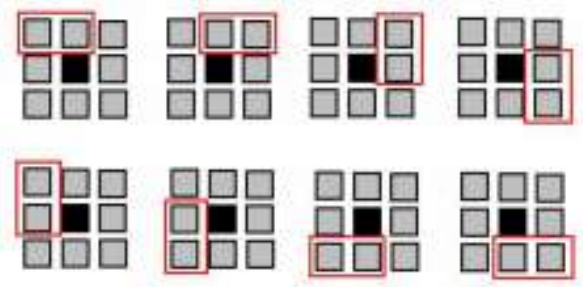

Fig 5. Eight sets of adjacent pixels pairs

The difference between two adjacent pixels is equal to one when they are not of the same color. Conversely, this difference is zero when two pixels are of the same color. The difference is individually computed for the eight sets of adjacent pixels illustrated in Figure 5. The eight differences are added together and the resultant sum is divided by two to compute crossing number for the black pixel at the center of the three-by-three pixel region. The center pixel corresponds to a termination minutia when the crossing number is equal to one. The center pixel is the location of a bifurcation when the crossing number is greater than or equal to three, and it is an intermediate ridge point when the crossing number is equal to two. Figure 6 illustrates intra-ridge pixels, termination minutia, and bifurcation minutia as detected by the crossing number.

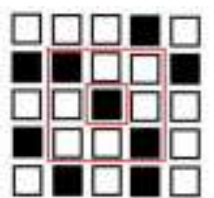

Cross no $=2$

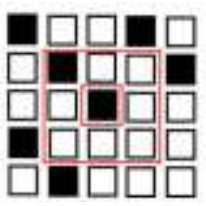

cross no $=1$

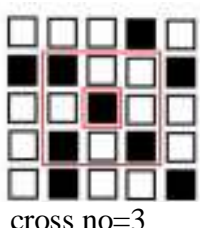

(c) Bifurcation

(a) Intra ridge pixel (b) Termination

Fig 6. Examples of crossing number.

The crossing number corresponding to each black pixel contained in the image is computed and used to delete unwanted spurs. The ridge is determined as unwanted spur if the trace reaches a bifurcation point in less than twenty pixels. On the other hand, if the trace length reaches twenty pixels before arriving at a bifurcation, the ridge is determined as valid ridge structure, and no change is made to the pixels as shown in Figure 7.

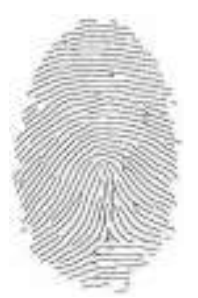

(a) Before removing spurs

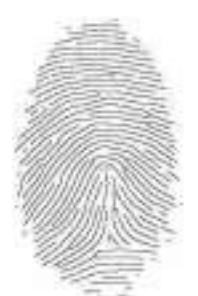

(b) After removing spurs Fig 7. Removing spurs.

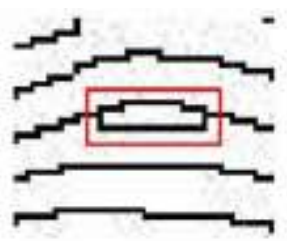

(a) Duplicate horizontal lines

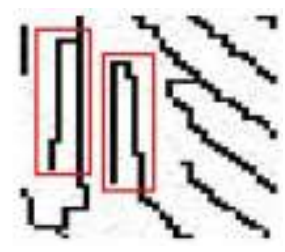

(b) Duplicate vertical lines Fig 8. Duplicate horizontal and vertical lines.

To remove the duplicate horizontal lines, the image is scanned for horizontal lines with a length of five pixels. Upon locating one of these lines, the five rows immediately beneath this line are analyzed. If one of these rows also has a line with a length of five pixels in the same column indices as the initial line detected, then it is classified as a duplicate horizontal line. Once all duplicate horizontal lines have been accounted for, the resulting spurs are deleted. The duplicate vertical lines are longer segments that only connect in one place, while the duplicate horizontal lines are short segments that connect to the ridge in two places. A duplicate vertical line exists when one of the columns has a line with a length of ten pixels in the same rows. At this point, the two vertical lines are traced downward. The trace continues until the ridge ends or a maximum trace length of forty pixels is reached, whichever comes first. As the traces are conducted, the total trace length is recorded for each segment. After the traces conclude, the total trace lengths for the two segments are compared. The vertical line that is part of the correct ridge structure does not come to a sudden end, and it should reach the maximum trace length. But the vertical line that is the unwanted segment will reach an end before reaching the maximum trace length. Thus, the segment that has the shorter trace length is determined as unwanted spur and is subsequently deleted.

\subsubsection{Final Noise Removal}

The noise produced by binarization and thinning is eliminated by identifying the short island segments near the outer boundary of the image. Starting at each termination the ridge is traced pixel by pixel and if another termination is reached within the length of seventeen pixels from the termination, it is considered as island segment and is deleted. If no termination is encountered until the maximum trace length is reached without any alterations, then that segment is retained. Figure 9 shows short island segments.

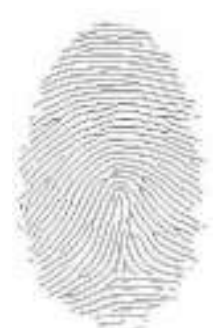

(a)Before

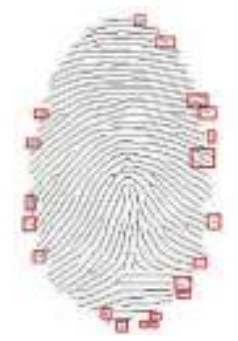

(b) Island segments to be deleted

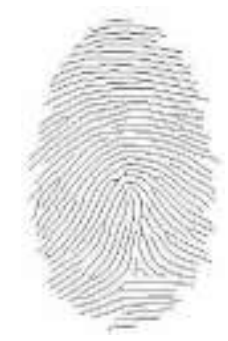

(c) After
Fig 9. Deleting short island segments

\subsubsection{Minutiae Extraction}

The duplicate horizontal and vertical lines may exist after removing the unwanted spurs, such as locations where a single ridge is represented by two horizontal lines or two vertical lines, as shown in Figure 8. 
In the minutia marking process the locations where the ridges end at the outer boundaries of the image are marked as terminations. However these locations are not unique termination minutiae. Instead, they only appear as terminations because the dimensions of the image make each ridge to come to an end. By creating an ellipse we can eliminate the minutia outside an ellipse and retain the inside minutia.

The center of the ellipse is established by locating the minimum and maximum rows and columns that contain a ridge pixel, then calculating the row and column that lie halfway between these extremes. The major axis of this ellipse is empirically selected as $94 \%$ of the difference between the minimum and maximum rows containing a ridge pixel, whereas the minor axis is empirically selected as $86 \%$ of the difference between the minimum and maximum columns with a ridge pixel. Figure10. Shows the ellipse generated for a fingerprint.

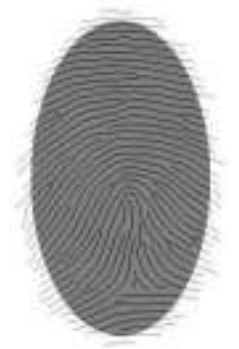

Fig 10. Ellipse to reject ridge endings along the boundaries.

A termination angle is the angle between the horizontal and the direction of the ridge, while a bifurcation angle is the angle between the horizontal and the direction of the valley ending between the bifurcations as shown in the Figure 11.
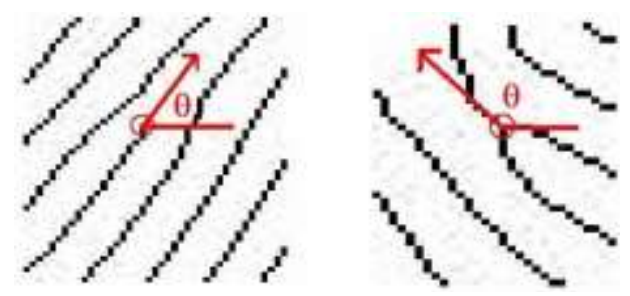

(a) Termination angle

(b) Bifurcation angle Fig 11. Definition of minutiae angles.

To compute the termination angles, the row and column indices for each termination are recorded. Beginning at each termination, the corresponding ridge is traced backwards by five pixels, and the resulting row and column indices are stored. In the plot, terminations are denoted by a square while bifurcations are displayed as diamonds as shown in figure 12.b

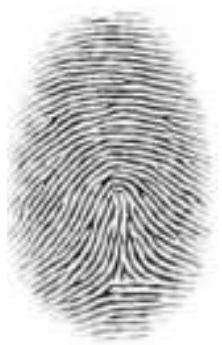

(a) Original image

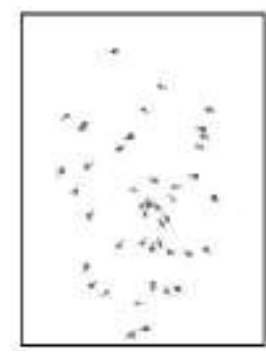

(b) Minutiae extracted

Fig 12. Minutiae detection

\subsubsection{False Minutia Removal}

The average inter-ridge width (D) refers to the average distance between two neighboring ridges. The procedure in removing false minutia is: (i) If the distance between one bifurcation and one termination is less than D and the two minutiae are in the same ridge, remove both of them. (ii) If the distance between two bifurcations is less than D and they are in the same ridge, remove the two bifurcations. (iii) If two terminations are within a distance $\mathrm{D}$ and their directions are coincident with a small angle variation, they satisfy the condition that no other termination is located between the two terminations, and then the two terminations are regarded as false minutia derived from a broken ridge, and are removed. (iv) If two terminations are located in a short ridge with length less than $\mathrm{D}$, remove the two terminations.

\subsubsection{Matching score}

The process involves comparing the set of minutiae data extracted from input image to a set of minutiae data extracted from template image. The matching begins by creating a matrix, called rotate values, of the orientation angle difference between each template minutiae, $T k(1 \leq k \leq N T)$, and each input minutiae, $\operatorname{Im}(1 \leq m \leq N I)$. NT and NI represent the total number of minutiae in the template and input image, respectively. The value at rotatevalues $(k, m)$ represents the difference between the orientation angles of $T k$ and $\mathrm{Im} . \mathrm{Tk}$ and Im represent the extracted data in all the columns of row $k$ and row $m$ in the template and input matrices, respectively. Template and input minutiae are selected as reference points for their respective data sets. Converting the template minutiae from row and column indices to polar coordinates are given in Equation 1.

$$
\left[\begin{array}{c}
r_{k}^{T} \\
\phi_{k}^{T} \\
\theta_{k}^{T}
\end{array}\right]=\left[\begin{array}{l}
\sqrt{\text { ow }_{k}^{T}-\operatorname{row}_{\text {ref }}^{T}-\boldsymbol{o l}_{k}^{T}-\operatorname{col}_{k}^{T}-} \\
\tan ^{-1}\left(\frac{\operatorname{row}_{k}^{T}-\operatorname{row}_{r e f}^{T}}{\operatorname{col}_{k}^{T}-\operatorname{col}_{\text {ref }}^{T}}\right) \\
\theta_{k}^{T}-\theta_{r e f}^{T}
\end{array}\right]
$$


Converting to polar coordinates allows for an effective match process conducted regardless of any rotational or translational displacement between the template and input images. The variable $r^{T}{ }_{k}$ represents the radial distance, $\varphi^{\mathrm{T}}{ }_{\mathrm{k}}$ represents the radial angle, and $\theta_{\mathrm{k}}^{T}$ represents the orientation of the $k^{\text {th }}$ minutia, all with respect to the reference minutiae. Also, the variables $\operatorname{row}_{k}^{T}$ and $\operatorname{col}^{T}{ }_{k}$ refer to the row and column indices of the $k^{\text {th }}$ minutia in the template matrix, while row $^{T}{ }_{\text {ref }}$ and $\mathrm{col}^{T}{ }_{\text {ref }}$ refer to the indices of the reference minutia currently being used for the template matrix. Equation 2 provides the polar coordinate transformation for the input minutiae.

$$
\left[\begin{array}{l}
r_{m}^{T} \\
\phi_{m}^{T} \\
\theta_{m}^{T}
\end{array}\right]=\left[\begin{array}{l}
\sqrt{\text { oow }_{m}^{T}-\operatorname{row}_{\text {ref }}^{T} \overline{\overline{2}}+\operatorname{ol}_{m}^{T}-\operatorname{col}_{k}^{T^{\overline{2}}}-} \\
\tan ^{-1}\left(\frac{\text { row }_{m}^{T}-\operatorname{row}_{\text {ref }}^{T}}{\operatorname{col}_{m}^{T}-\operatorname{col}_{\text {ref }}^{Y}}\right)+\text { rotatevalues } \boldsymbol{k}, m \\
\theta_{m}^{T}-\theta_{\text {ref }}^{T}
\end{array}\right]
$$

Instead of comparing row and column indices, the comparison is now based on the relative position of the minutiae with regards to the reference minutiae. The relative positions of the minutiae remain the same even as the absolute position of the fingerprint image changes. The difference between the template and input data is computed for the radial distance, radial angle, and orientation angle. Two minutiae are determined to match if (i) the absolute radial distance difference is less than a tolerance of three pixels. (ii) The absolute radial angle difference is less than a tolerance of five degrees. (iii) The absolute orientation angle difference is less than a tolerance of ten degrees. (iv) The two minutiae are the same type. A matching score representing the similarity between the datasets is calculated for each combination of reference points using the total number of minutiae matched in each case. Equation 3 shows the formula for calculating the matching score when $T k$ and $I m$ are being used as reference.

Minutiae matching score $(k, m)=$

\section{Number of minutiae pairs matched}

$$
\operatorname{MAX}(\mathrm{Mi}, \mathrm{Mt})
$$

Where Mi and Mt represents the Total number of minutiae in input image and Total number of minutiae in template image. By this definition, the matching score takes on a value between zero and one. A matching score of one indicates the data matches perfectly, whereas a matching score of zero occurs when there are no matching minutiae. Matching scores are calculated for every possible combination of reference points and stored in the matching score matrix. The final matching score between the template and input datasets is selected as the maximum value in the matching score matrix. The fingerprint is matched if the maximum score is more than threshold whereas fingerprint is not matched if the maximum score is less than threshold.

\subsubsection{Ridge Extraction using Hough Transform}

Hough transform is used to detect curves in images, and in particular, is used for straight-line detection. Hough transform algorithm requires an accumulator array whose dimension corresponds to the number of parameters of the curve being detected.
In the case of straight lines, since the equation $y=a x+b$ as unbounded parameters, the equation $\mathrm{r}=x \cos \mathrm{q}+y \sin \mathrm{q}$ is generally used where $r$ is the perpendicular distance from the origin to the straight line and $\mathrm{q}$ is the angle made by the perpendicular with the $x$-axis. So, for the straight-line detection, it is necessary to use a two dimensional accumulator array. The extracted straight lines that better matches a ridge are shown in Figure 13.

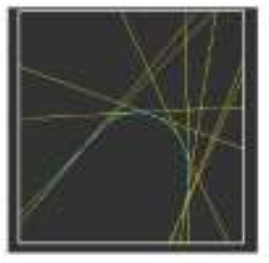

Fig 13. Straight lines detected from a given (highlighted) fingerprint ridge by using the Hough transform.

Hough line function gives three parameters Hough space accumulator, a vector that contains radius of detected lines in polar coordinates system and a vector that contains angle of detected lines in polar coordinate system. Matching score of ridge based technique is computed by the following formula given in the Equation 4.

Ridge matching score $=$

\section{Number of lines matched $\operatorname{MAX}(\mathrm{Li}, \mathrm{Lt})$}

Where $\mathrm{Li}$ and $\mathrm{Lt}$ Represents the total number of straight lines detected in the input image and total number of lines detected in template image respectively.

\subsubsection{Minutiae and Ridge Score Fused using Strength Factors}

The minutiae and ridge matching scores are combined using Equation 5.

$$
\begin{aligned}
& \mathrm{HMS}=\alpha \mathrm{Sr}+\beta^{2} \mathrm{Sm} \text {---------- (5) } \\
& \alpha+\beta=1 \text {---------------------- (5) } \\
& \text { Where } \mathrm{Sr}=\text { Ridge Matching Score } \\
& \mathrm{Sm}=\text { Minutiae Matching Score } \\
& \text { HMS }=\text { Hybrid Matchning Score } \\
& \alpha \text { and } \beta \text { are strength factors }
\end{aligned}
$$

The matching percentage value of two different Fingerprints is low compared to the existing algorithms for every value of $\alpha$ and $\beta$.

\section{ALGORITHM}

Problem definition: The Fingerprint is verified using Fingerprint Verification based on fusion of Minutiae and Ridges using Strength Factors.

Objectives of the algorithm are:

(i) The Minutiae Extraction using thinned image by Block Filter. 
(ii) The true ridge information is extracted by Hough Transform.

(iii) The true minutiae and ridges information are combined to get matching score.

Assumption: Data base of fvc2004 [18] is considered for performance analysis.

\section{Table 1 Algorithm of FVMRSF}

- Input : Fingerprint Image

- $\quad$ Output: Verified Fingerprint Image

1. Binarizing the gray scale fingerprint image.

2. Ridge thickness of binarized image is reduced to 1-pixel width by thinning of the image by Block Filter.

3. Minutiae extraction is done on thinned image by considering the region of interest by using an Ellipse

4. False Minutiae removal.

5. Extracting the ridge information of the image by Hough Transform.

6. Compute the minutiae and ridge matching scores.

7. Compute the hybrid matching score.

$\mathrm{HMS}=\alpha \mathrm{Sr}+\beta^{2} \mathrm{Sm}$

The algorithm of FVMRSF is given in the Table 1, in which the Fingerprint is verified using the combination of minutiae and ridge data with Strength Factors.

\section{PERFORMANCE ANALYSIS}

The Fingerprint data base of fvc2004 is considered for the performance analysis. The performance of minutiae technique is poor for low quality fingerprint images such as small area of overlap between the template and query image. The ridge based method has disadvantage compared to minutiae technique as the number of parameters for matching are less, hence it may reject genuine fingerprint. The combination of both minutiae and ridge based technique improves the performance results by eliminating disadvantages of each method. In the existing Hybrid Fingerprint Matcher (HFM) method, the minutiae matching scores are computed with two techniques of minutiae extraction.

In the proposed FVMRSF algorithm the minutiae and ridges are extracted using Block Filter and Hough Transform respectively. The minutiae and the ridge parameters are fused using the Strength Factors in our algorithm to improve the performance compared to the existing algorithm. Table 2 gives the matching percentage of two different fingerprints for four samples. It is observed that the FVMRSF technique gives better results compared to minutiae technique, ridge technique and HFM.
The proposed method has percentage improvement in the range of 10 to 54 compared to the existing HFM method when two different Fingerprints are compared. The matching percentages of two similar fingerprints for four samples are given in Table 3. and is $100 \%$ for all four methods.

Table 2 Matching percentages of two different Fingerprints

\begin{tabular}{|c|c|c|c|c|}
\hline & $\begin{array}{l}\text { Ridge } \\
\text { Technique }\end{array}$ & $\begin{array}{l}\text { Minutiae } \\
\text { Technique }\end{array}$ & HFM & FVMRSF \\
\hline Sample1 & 10 & 8.875 & 9.437 & 8.474 \\
\hline Sample2 & 11.11 & 8.7558 & 9.932 & 7.743 \\
\hline Sample3 & 7.142 & 8.8496 & 7.995 & 5.783 \\
\hline Sample4 & 8.333 & 12.335 & 10.33 & 7.250 \\
\hline
\end{tabular}

Table 3 Matching percentage of two similar fingerprints

\begin{tabular}{|l|c|c|c|c|}
\hline & $\begin{array}{l}\text { Ridge } \\
\text { Technique }\end{array}$ & $\begin{array}{l}\text { Minutiae } \\
\text { Technique }\end{array}$ & HFM & FVMRSF \\
\hline Sample1 & 100 & 100 & 100 & 100 \\
\hline Sample2 & 100 & 100 & 100 & 100 \\
\hline Sample3 & 100 & 100 & 100 & 100 \\
\hline Sample4 & 100 & 100 & 100 & 100 \\
\hline
\end{tabular}

\section{CONCLUSION}

Fingerprint verification is most reliable characteristics for personal identification as it is unique and persistent. In this paper we proposed Fingerprint Verification based on fusion of Minutiae and Ridges using Strength Factors in which the minutiae and ridge methods are combined. The Block Filter and Hough Transform are used to extract minutiae and ridges respectively. The FVMRSF gives better results for different fingerprint matching compared to existing algorithms. In Future the Wavelet Transform is introduced to improve the results further.

\section{REFERENCES}

[1] M. Kucken and A. C. Newell, "Fingerprint Formation," Proceedings of Journal of Theoretical Biology, pp. 71-83, 2005.

[2] Ashish mishra and Madhu Shandilya, "Fingerprint Core Point Detection using Gradient Field Mask," International Journal of Computer Applications, vol 2, pp19-23, 2010.

[3] Bazen and Gerez, "Extraction of Singular Points from Directional Fields of Fingerprints," Annual Centre for Telematics and Information Technology Workshop, vol 24, pp 905-919, July 2002.

[4] E. K. Yun and S. B. Cho, "Adaptive Fingerprint Image Enhancement with Fingerprint Image Quality Analysis," International conference of Image and Vision Computing, pp. 101-110, 2006.

[5] M. P. Brankica and L. Maskovic, "Fingerprint Minutiae Filtering Based on Multiscale Directional Information," FACTA Universitatis-Series: Electronics and Energetics, vol. 20, pp.233-244, August 2007.

[6] F. A. Afsar, M. Arif and M. Hussain, "Fingerprint Identification and Verification System using Minutiae Matching," National Conference on Emerging Technologies, pp.141-146, 2004. 
[7] G. Jagadeeswar Reddy, T. Jaya Chandra Prasad and M. N. Giri Prasad, "Fingerprint Image Denoising using Curvelet Transform," Proceedings of Asian Research Publishing Network Journal of Engineering and Applied Sciences, vol 3, no 3, pp. 31-35, June 2008.

[8] K. Zebbiche and F. Khelifi, "Region-Based Watermarking of Biometrics Images: Case Study in Fingerprint Images," Proceedings of International Journal of Digital Multimedia Broadcasting, pp. 1-13, 2008.

[9] Bhupesh Gour, T. K. Bandopadhyaya and Sudhir Sharma, "Fingerprint Feature Extraction using Midpoint Ridge Contour Method and Neural Network," Proceedings of International Journal of Computer Science and Network Security, vol.8, no.7, pp. 99-103, July 2008.

[10] Seung-Hoon Chae and Jong $\mathrm{Ku}$ Kim "Ridge-Based Fingerprint Verification for Enhanced Security," Digest of Technical Papers International Conference on Consumer Electronics, pp 1-2, 2009.

[11] A. N. Marana, and A. K. Jain, "Ridge-Based Fingerprint Matching using Hough Transform," IEEE Proceedings of the Brazilian Symposium on Computer Graphics and Image Processing, pp. 112-119, October 2005.

[12] A. K. Jain, S. Prabhakar and A. Chen, "Combining Multiple Matchers for a High Security Fingerprint Verification System," Pattern Recognition Letters, Elsevier Science Direct, vol. 20, pp 1371- 1379, 1999.

[13] Fanglin Chen and Jie Zhou, "Reconstructing Orientation Field from Fingerprint Minutiae to Improve Minutiae-Matching Accuracy," IEEE Transactions on image processing, vol. 18, no 4, pp 1665-1670, July 2009.

[14] Chunxiao Ren and Yilong Yin, "A Linear Hybrid Classifier for Fingerprint Segmentation," Fourth International Conference on Neural Computation, pp 33-37, November 2008.

[15] Hartwig Fronthaler and Klaus Kollreider, "Local Features for Enhancement and Minutiae Extraction in Fingerprints," IEEE Transactions on Image Processing, vol. 17, no 3, pp 354-363, 2008.

[16] Shabana Tadvi and Mahesh Kalte, "A Hybrid System for Fingerprint Identification," International Journal of Computer Science and Engineering, vol 2, no 3, pp 767-771, 2010.
[17] Liu Wei and Zhou Cong, "Efficient Gradual Segmentation of Fingerprint Images," Proceedings of Sixth WSEAS International Conference on Multimedia Systems and Signal Processing, pp 67-70, 2006.

[18] Fingerprint Verification Competition, Database http://bias.csr.unibo.it/fvc2004.

[19] Hemanth Krishnappa and Hongyu Guo, "Fingerprint Verification using Mutual Information," Journal of The Consortium for Computing Sciences in Colleges, 15-22,2008.

[20] Swapnali Mahadik, K Narayanan, D V Bhoir and Darshana Shah, " Access Control System using Fingerprint Recognition," International Conference on Advances in Computing, Communication and Control, pp 306-311, 2009.

[21] Yi Chen and A K Jain, "Beyond Minutiae: A Fingerprint Individuality Model with Patteren, Ridge and Pore Features," International Conference on Biometrics, pp 523-533, 2009.

[22] Jong KU Kin, Seung-Hoon Chae, Sung Jin Lim and Sung Bum Pan, "A Study on the Performance Analysis of Hybrid Fingerprint Matching Methods," International Journal of Future Generation Communication and Networking, pp 23-28, 2008.

[23] Manvjeet Kaur, Mukhwinder Singh, Akshay Giridhar and Parvinder S. Sandhu, "Fingerprint Verification System using Minutiae Extraction Technique," Proceedings of World Academy of Science, Engineering and Technology, vol 36, pp 497-502, 2008.

[24] Liu Wei, "Fingerprint Classification using Singularities Detection," International Journal of Mathematics and Computers in Simulation, vol 2, issue 2, pp 158-162, 2008.

[25] Arun Ross, Anil Jain and James Reisman, "A Hybrid Fingerprint Matcher," Proceedings of International Conference on Pattern Recognition, pp 1661-1673, 2003.

[26] Zhang yuanyuan and Jing Xianjun, "Spectral Analysis Based Fingerprint Image Enhancement Algorithm," International Conference on Image Analysis and Signal Processing. pp 656659, 2010. 